Sociologie et sociétés

\title{
Liste des personnes ayant préparé des évaluations de manuscrits pour Sociologie et sociétés
}

Volume 30, numéro 2, automne 1998

Le syndicalisme

URI : https://id.erudit.org/iderudit/001448ar

DOI : https://doi.org/10.7202/001448ar

Aller au sommaire du numéro

Éditeur(s)

Les Presses de l'Université de Montréal

\section{ISSN}

0038-030X (imprimé)

1492-1375 (numérique)

Découvrir la revue

Citer ce document

(1998). Liste des personnes ayant préparé des évaluations de manuscrits pour Sociologie et sociétés. Sociologie et sociétés, 30(2), 231-234.

https://doi.org/10.7202/001448ar d'utilisation que vous pouvez consulter en ligne. 


\section{Liste des personnes ayant préparé des évaluations de manuscrits pour Sociologie et sociétés*}

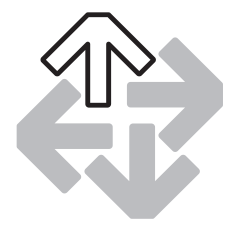

La direction tient à exprimer sa plus vive reconnaissance aux collègues qui ont bien voulu accepter d'évaluer un ou plusieurs manuscrits soumis à la revue, en vue des numéros de 1996-1997 et 1997-1998.

\section{6-1997}

M. Barry D. ADAM, Department of Sociology and Anthropology, University of Windsor Mme Mary Louise ADAMS, Department of Sociology, Carleton University

M. Douglas E. ANGUS, Programme MHA, Faculté d'administration, Université d'Ottawa

M. Rachad ANTONIUS, Centre d'études ethniques, Université de Montréal Mme Patricia BAIRD, Department of Medical Genetics, The University of British Columbia M. Renaldo N. BATTISTA, Service d'épidémiologie clinique, Hôpital général de Montréal M. Léon BERNIER, INRS-Culture et société, Montréal

Mme Isabelle BERTAUX-WIAME, CNRS-Travail et mobilités, Université Paris X

M. Gilles BIBEAU, Département d'anthropologie, Université de Montréal

M. Guy BOURGEAULT, Département d'études en éducation et d'administation de l'éducation, Université de Montréal

Mme Michèle BOURGON, Département de travail social, Université du Québec à Montréal

M. Michel CLAES, Département de psychologie, Université de Montréal

M. Olivier CLAIN, Département de sociologie, Université Laval

M. Jean-François CÔTÉ, Département de sociologie, Université du Québec à Montréal

M. André-Pierre CONTANDRIOPOULOS, Département d'administration de la santé, Université de Montréal

Mme Denise COUTURE, Département de sociologie, Université de Montréal

Mme Denise COUTURE, Faculté de théologie, Université de Montréal

Mme Andrée DEMERS, Groupe de recherche sur les aspects sociaux de la santé et de la prévention, Département de sociologie, Université de Montréal 
Mme Francine DESCARRIES, Département de sociologie, Université du Québec à Montréal

M. Michel DE SÈVE, Département de sociologie, Université Laval

M. Jean DORION, Délégation générale du Québec, Tokyo

M. Jules DUCHASTEL, Département de sociologie, Université du Québec à Montréal

Mme Brigitte DUMAS, Département de sociologie, Université de Montréal

Mme Lise DUNNIGAN, Service de l'évaluation des politiques et des programmes, ministère de la Santé et des Services sociaux, Gouvernement du Québec

Mme Undine DU PLESSIS, Comparative Literature and Humanities, University of Colorado at Boulder

M. Gilles DUSSAULT, Département d'administration de la santé, Université de Montréal

M. Robert G. EVANS, Centre for Health Services and Policy Research, University of British Columbia

Mme Andrée FORTIN, Département de sociologie, Université Laval

M. Clarke FRASER, Département de génétique humaine, Université McGill

M. Gabriel GAGNON, Département de sociologie, Université de Montréal

Mme Mona-Josée GAGNON, Département de sociologie, Université de Montréal

M. Jacques T. GODBOUT, INRS-Urbanisation, Montréal

Mme Céline GOULET, Faculté des sciences infirmières, Université de Montréal

Mme Line GRENIER, Département de communication, Université de Montréal

M. Hubert GUINDON, Département de sociologie et d'anthropologie, Université Concordia

Mme Ratiba HADJ-MOUSSA, Women's Studies, Queen's University

M. Joseph HOFBECK, Théologie, Université Concordia

M. David HOWES, Département de sociologie et d'anthropologie, Université Concordia

M. Roch HURTUBISE, Département de service social, Université de Sherbrooke

M. Claude LABERGE, Département de génétique humaine, CHUL, Sainte-Foy

Mme Danielle LABERGE, Département de sociologie, Université du Québec à Montréal

M. Camille LEGENDRE, Département de sociologie, Université de Montréal

Mme Diane LAMOUREUX, Département de science politique, université Laval

M. Jean-Marie LANCE, Conseil d'évaluation des technologies de la santé du Québec, Montréal

Mme Isabelle LASVERGNAS, Département de sociologie, Université du Québec à Montréal

Mme Nicole LAURIN, Département de sociologie, Université de Montréal

Mme Gisèle LEGAULT, École de service social, Université de Montréal

Mme Thérèse LEROUX, Centre de recherche en droit public, Faculté de droit, Université de Montréal Mme Abby LIPPMAN, Department of Epidemiology and Biostatistics, Université McGill

M. Maurice McGREGOR, Conseil d'évaluation des technologies de la santé du Québec, Montréal

M. Guy MÉNARD, Département de sciences religieuses, Université du Québec à Montréal

Mme Jacinthe MICHAUD, Programme d'études des femmes, collège Glendon

Madame Pauline MORISSETTE, École de service social, Université de Montréal

Mme Louise NADEAU, Département de psychologie, Université de Montréal

Mme Françoise-Romaine OUELLETTE, INRS - Culture et société, Montréal

Mme Simone NOVAES, Centre de recherche Sens, Éthique, Société, CNRS, Paris

M. Holger A. PAUSCH, Department of Modern Languages and Comparative Studies, University of Alberta

M. Michel PERREAULT, Faculté des sciences infirmières, Université de Montréal

Mme Louise POTVIN, Groupe de recherche interdisciplinaire en santé, Faculté de médecine, Université de Montréal

M. Luc RACINE, Département de sociologie, Université de Montréal

M. Marcel RAFIE, Département de sociologie, Université du Québec à Montréal

M. Gilbert RENAUD, École de service social, Université de Montréal

M. Guy ROCHER, Centre de recherche en droit public, Faculté de droit, Université de Montréal

Mme Marie-Andrée ROY, Département de sciences religieuses, Université de Montréal 
M. Stephen SCHECTER, Département de sociologie, Université du Québec à Montréal

M. Éric SCHWIMMER, Département d'anthropologie, Université Laval

M. Robert SÉVIGNY, Département de sociologie, Université de Montréal

M. John STOUT, Department of French, McMaster University

Mme Marie-Blanche TAHON, Département de sociologie, Université d'Ottawa

Mme Catherine TAYLOR, Centre for Academic Writing, University of Winnipeg

M. Jean-Guy VAILLANCOURT, Département de sociologie, Université de Montréal

M. Thomas WAUGH, Department of Cinema, Université Concordia

\section{$1997-1998$}

M. Benigno E. AQUIRRE, Department of Sociology, Texas A \& M University

M. Claude BARITEAU, Département d'anthropologie, Université Laval

M. Philippe BATAILLE, Centre d'analyse et d'intervention sociologiques, École des hautes études en sciences sociales, Paris

Mme Bernadette BAWIN-LEGROS, Faculté d'économie, de gestion et de sciences sociales, Université de Liège

M. Pierre W. BÉLANGER, Département d'orientation, d'administration et d'évaluation en éducation, université Laval

M. Léon BERNIER, INRS-Culture et société, Montréal

M. Christian BERTAUX, Laboratoire de changement social, Université Paris VII-Denis Diderot

M. Gérard BOISMENU, Département de science politique, Université de Montréal

M. Jean-Paul BRODEUR, École de criminologie, Université de Montréal

M. Robert BRYM, Department of Sociology, University of Toronto

M. Jean-François CHANLAT, Service de l'enseignement de la direction et de la gestion des organisations, École des Hautes Études commerciales, Montréal

Mme Yolande COHEN, Département d'histoire, Université du Québec à Montréal

M. André CORTEN, Département de science politique, Université du Québec à Montréal

M. Robert CRÉPEAU, Département d'anthropologie, Université de Montréal

Maria DE KONINCK, Département de médecine sociale et préventive, Université Laval

M. Roger DE LA GARDE, Département d'information et de communication, Université Laval

M. Françcois DE SINGLY, Département de sciences sociales, Université René Descartes Paris V

Mme Brigitte DUMAS, Département de sociologie, Université de Montréal

Mme Micheline DUMONT, Département d'histoire et de sciences politiques, Université de Sherbrooke

M. Richard FOURNIER, ministère de l'Éducation, Gouvernement du Québec

M. Gilles GAGNÉ, Département de sociologie, Université Laval

M. Jean-Claude GARDIN, CNRS, Paris

M. Robert GIROUX, Département de littérature, Université de Sherbrooke

Mme Line GRENIER, Département de communication, Université de Montréal

M. Yves GOUSSAULT, Institut d'étude du développement économique et social, Université de Paris I-Panthéon-Sorbonne

M. Louis GUAY, Département de sociologie, Université Laval

M. Hubert GUINDON, Département de sociologie et d'anthropologie, Université Concordia

M. Jacques HAMEL, Département de sociologie, Université de Montréal

M. Fernand HARVEY, INRS-Culture et société, Québec

M. Ross HASTINGS, Département de criminologie, Université d'Ottawa

M. Roch HURTUBISE, Département de service social, Université de Sherbrooke

M. John JACKSON, Département de sociologie et d'anthropologie, Université Concordia

M. Bogumil JEWSIEWICKI, Département d'histoire, Université Laval

M. Pierre LANDREVILLE, École de criminologie, Université de Montréal

Mme Anne LAPERRIÈRE, Département de sociologie, Université du Québec à Montréal 
M. Camille LEGENDRE, Département de sociologie, Université de Montréal

Mme Denise LEMIEUX, INRS-Culture et société, Montréal

M. Christopher McALL, Département de sociologie, Université de Montréal

M. Roberto MIGUELEZ, Département de sociologie, Université d'Ottawa

M. Walter MOSER, Département de littérature comparée, Université de Montréal

Mme Anne MUXEL, Centre d'étude de la vie politique française, Fondation nationale des sciences politiques, Paris

M. Greg NIELSEN, Département de sociologie, Université Concordia

M. Alain NÖEL, Département de science politique, Université de Montréal

M. Émile OLLIVIER, Département d'études en éducation et d'administration de l'éducation, Université de Montréal

M. Jean-Claude PASSERON, École des hautes études en sciences sociales, Paris

M. Jacques PELLETIER, Département d'études littéraires, Université du Québec à Montréal

M. André PETITAT, Faculté des sciences sociales et politiques, Université de Lausanne

M. Alvaro PIREZ, Département de criminologie, Université d'Ottawa

Mme Agnès PITROU, Laboratoire d'économie et de sociologie du travail, CNRS, Aix-en-Provence Mme Elspeth PROBYN, Women's Studies Centre, The University of Sydney

M. Bill REIMER, Département de sociologie et d'anthropologie, Université Concordia

M. Serge ROBERT, Département de philosophie, Université du Québec à Montréal

Mme Régine ROBIN, Département de sociologie, Université du Québec à Montréal

M. Guy ROCHER, Centre de recherche en droit public, Faculté de droit, Université de Montréal

M. Michael R. SMITH, Département de sociologie, Université McGill

M. J. Yvon THÉRIAULT, Département de sociologie, Université d'Ottawa

M. Michel TRÉPANIER, INRS-Urbanisation, Montréal

M. Henrique URBANO, Département de sociologie, Université Laval

M. Jean-Guy VAILLANCOURT, Département de sociologie, Université de Montréal

Mme Louise VANDELAC, Département de sociologie, Université du Québec à Montréal 